\title{
SEMANTIC INTEGRATION OF MULTIPLE HEALTH DATA FOR TREATMENT DECISION-MAKING IN LOW-RESOURCE SETTINGS
}

\author{
Olawande Daramola ${ }^{1}$ and Thomas Moser $^{2}$

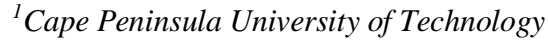 \\ PO Box 652 Cape Town 8000 | Corner of Keizergracht and Tennant Street, Zonnebloem \\ South Africa \\ ${ }^{2}$ St. Pölten University of Applied Sciences, Matthias Corvinus-Straße 15, 3100 St. Pölten \\ Austria
}

\begin{abstract}
Accurate treatment decision-making for disease treatment is important, particularly if it is done in a way that helps to overcome the challenges associated with low resource setting. This include shortage of qualified personnel, infrastructure, ready access to devices, and healthcare by common people. Although real-time gait analysis has been used for the diagnosis of diseases that are associated with gait impairments, the need to improve on the performance of gait-based prediction by augmenting it with other sources of knowledge in a complementary way has been acknowledged. In contrast, to existing approaches, this paper presents the design of a conceptual framework that will enable the semantic integration and use of information from multiple health data sources in order to ensure efficient treatment decision making for gait-related diseases in a low resource setting such as South Africa. The analytical evaluation of the proposed framework suggests that it has sufficient merit and potential to be usable and useful in low-resource settings.
\end{abstract}

\section{KEYWORDS}

Low Resource Settings, Semantic Integration, Health Data Integration, Electronic Health Records, Disease Treatment, Decision Making

\section{INTRODUCTION}

Accurate and prompt treatment decision-making by medical practitioners in dealing with patients is critical to ensuring the health and well-being of patients. It is particularly important when this is done in low resource settings where there are myriad of challenges that impedes good healthcare conditions. Some of the challenges of low resource settings, which any viable healthcare solution must overcome include shortage of qualified personnel, lack of good infrastructure, lack of ready access to mobile devices, and high cost of healthcare for common people (Fritz et al., 2015; Zargaran et al., 2014). The advent of new digital technologies such as cloud computing, mobile computing, wearable sensors, and linked open data has created new opportunities for expert decision-making in terms of disease diagnosis, disease treatment, and patient's rehabilitation - irrespective of the physical distance between the patient and the medical practitioner.

Gait analysis (GA), which is also known as locomotive analysis entails collecting quantitative data on the pattern of physical movements of a human in order to understand the etiology of gait defects and the formulation of an appropriate treatment plan. GA has been used as a basis for disease diagnosis for patients with walking impairment, and the treatment of many neurological disorders and cardiovascular diseases (Tang and Su, 2013). Currently, it is possible to obtain real-time information on a patient's gait pattern while the patient is walking both within and outside the hospital environment. Critical gait parameters such as gait speed, cadence, step length, stride length and many more can be measured by using wearable body sensors that are attached to either knees, using sensor-insoles for shoes, or sensors for legs and limbs (Horak et al., 2015; Horsak et al., 2016). These gait parameters have been associated with the symptoms of some specific diseases, which make them suitable as a basis for disease diagnoses, disease treatment, gait retraining, and 
gait rehabilitation of patients that are being treated for gait impairment. However, real-time gait analysis is still confronted with several challenges, which include the need to i) improve the precision of diseases diagnosis based on gait factors; ii) adapt real-time gait analysis for application in resource-constrained settings; iii) identify specific gait parameters that are more associated with specific types of diseases; and iv) engender gait improvement during gait retraining and gait rehabilitation by using multisensory feedback mechanism (Tang and Su, 2013; Zhou et al., 2012; Zillner and Sonntag, 2012). Hence, there is the need to augment real-time gait analysis predictions with knowledge from other sources in order to enhance its efficiency (Horak et al., 2015; Zhou et al., 2012; Zillner and Sonntag, 2012). It is particularly more challenging to attain efficient treatment decision-making for gait-related diseases in low resource settings.

This paper, by focusing on gait-related diseases, presents the conceptual design of a framework (SemTreat) that leverages the semantic integration of data from multiple health data sources such as real-time gait analysis data, linked electronic health records (EHR), epidemiological data, and other open data sources in order to facilitate decision making for disease diagnosis and disease treatment in low resource settings . It is expected that the provision of our type of proposed framework will enable i) medical practitioners to make more informed decisions on the treatment of patients based on their medical information; ii) patients to have continuation of their treatment if they happen to change their location/city of residence; and iii) patients to identify the type of diseases that they might have, and make a decision on the appropriate self-management strategies that could be adopted.

The rest of this paper is organized as follows. Section 2 presents the background and related work, while Section 3 gives an overview of the methodology and design adopted for the SemTreat framework. Section 4 describes the approaches that will be used for the evaluation of the proposed framework. The paper is concluded in Section 5 with a brief note and overview of future work.

\section{BACKGROUND AND RELATED WORK}

This section presents an overview of related work in the area of real-time gait analysis, and background on electronic health records (EHR), and semantic integration.

So far, many applications of gait analysis have been reported in the literature, particularly to aid the treatment of diseases that are associated gait impairment such as neurological disorders and cardiovascular diseases (Horak et al., 2015; Tang and Su, 2013). Also, it has been used in conjunction with sonification for the purpose of gait training and gait rehabilitation (Effenberg et al., 2011; Rodger et al., 2014). However, most of these systems have been deployed within the laboratory setting, which makes them unable to support everyday use outside the hospital environment. A few of the exceptions to this are reported in (Noehren et al., 2011; Redd and Bamberg, 2012), but even then, these systems were not been designed or deployed in low resource settings, hence the ability of these real-time gait analysis systems to function in low resource settings has not be verified. Low cost and affordable sensor technologies for gait-data acquisition are required in order to facilitate real-time gait analysis. Another issue is the need to improve the accuracy of gait measures as a basis for disease diagnosis because gait factors are dependent on other external factors such as emotions and environment. Hence, the need to augment gait prediction with other sources of health data such as EHR and open linked data.

Electronic health records (EHR) are repositories of personal medical information of individuals. EHR provide a rich source of information on a patient including pathology data, family history, medical history, primary care data, and secondary care data (Lobo et al., 2017; Zhao and Weng, 2011). If accessible, EHR can be integrated with real-time gait data as a basis for accurate disease diagnosis, and formulation of a suitable treatment plan for a patient. Recently, the instances of the use of EHR for research purposes have been on the increase. Examples of these include CALIBER (Morley et al., 2014), HealthID ${ }^{1}$, epSOS (Fonseca et al., 2015), openEHR ${ }^{2}$, HEOR (Asaria et al., 2016), semantichealthnet (Martinez-Costa et al., 2014). Many of the EHR approaches focussed on the interoperability of EHR (Adebesin et al., 2013; Adenuga et al., 2015; Fonseca et al., 2015; Martinez-Costa et al., 2014), and the use of EHR for the purpose of informed decision

\footnotetext{
${ }^{1}$ HealthID: the technology that puts your patients' health records in your hands. available at: https://www.discovery.co.za/medical-aid/health-id

${ }^{2}$ OpenEHR. An open domain-driven platform for developing flexible e-health systems. http://www.openehr.org
} 
making on patient`s treatment (Asaria et al., 2016; Lobo et al., 2017; Morley et al., 2014; Zhao and Weng, 2011).

Semantic integration enables meaningful communication between different terms that have been used for similar domains concepts. It enables diverse systems to understand one another and engage in meaningful conversation (Aldred et al., 2006; Doan and Halevy, 2005; Hohpe, 2006; Moser and Biffl, 2012; Noy, 2004). In contrast to existing approaches, this research will explore the semantic integration of real-time gait data, EHR, epidemiological data, open linked data, and other open data sources for treatment decision making in low resource settings for some diseases that are associated with gait impairment.

\section{METHODOLOGY AND DESIGN}

This section starts with describing the requirements of the proposed SemTreat framework as well as the included stakeholders, then continues with defining the proposed architecture of the SemTreat framework, and finally identifies potential use case scenarios for the usage of the SemTreat framework.

\subsection{SemTreat Requirements Elicitation}

The requirements were gathered in order to understand both the technical as well as the user requirements for the semantic integration of linked EHR, epidemiological data, and real-time gait data. This was done by the help of a requirements analyst, medical experts, and epidemiologists in order to elicit knowledge from the domain experts and relevant stakeholders. During the requirements elicitation process, the following stakeholders of the SemTreat framework were identified: medical practitioners, patients, and governmental organizations.

The requirements of the SemTreat framework are structured into the following categories:

- practicability of the SemTreat framework

- centralized overview of distributed health data of a single patient: should be usable both by the patient as well as by medical practitioners that have been granted such authorized access

- functionality in low-resource settings

- limited connectivity regarding both signal strength as well as bandwidth electrical power availability;

- Accessibility of technology to patients in terms of cost of devices and internet data;

- usability of the SemTreat frameworks application (mobile)

$\circ \quad$ smartphone vs. regular mobile phone version

- usage should also be possible (in a limited way) with low literacy as well as low technical affinity (e.g. elderly persons)

- $\quad$ privacy and data protection

$\circ$ personal health data is very sensitive information and needs to be well protected

- each patient should be able to define for which other parties he/she wants to share the personal health data

- aggregated but anonymized health data should be available for usage by governmental organizations, e.g. for statistical analysis, data analytics, and strategic planning

\subsection{Description of the SemTreat Architectural Design}

The SemTreat architecture is conceptualized in way that makes it suitable to facilitate the envisioned capability to aid treatment decision making on gait-related diseases in low resource settings. 


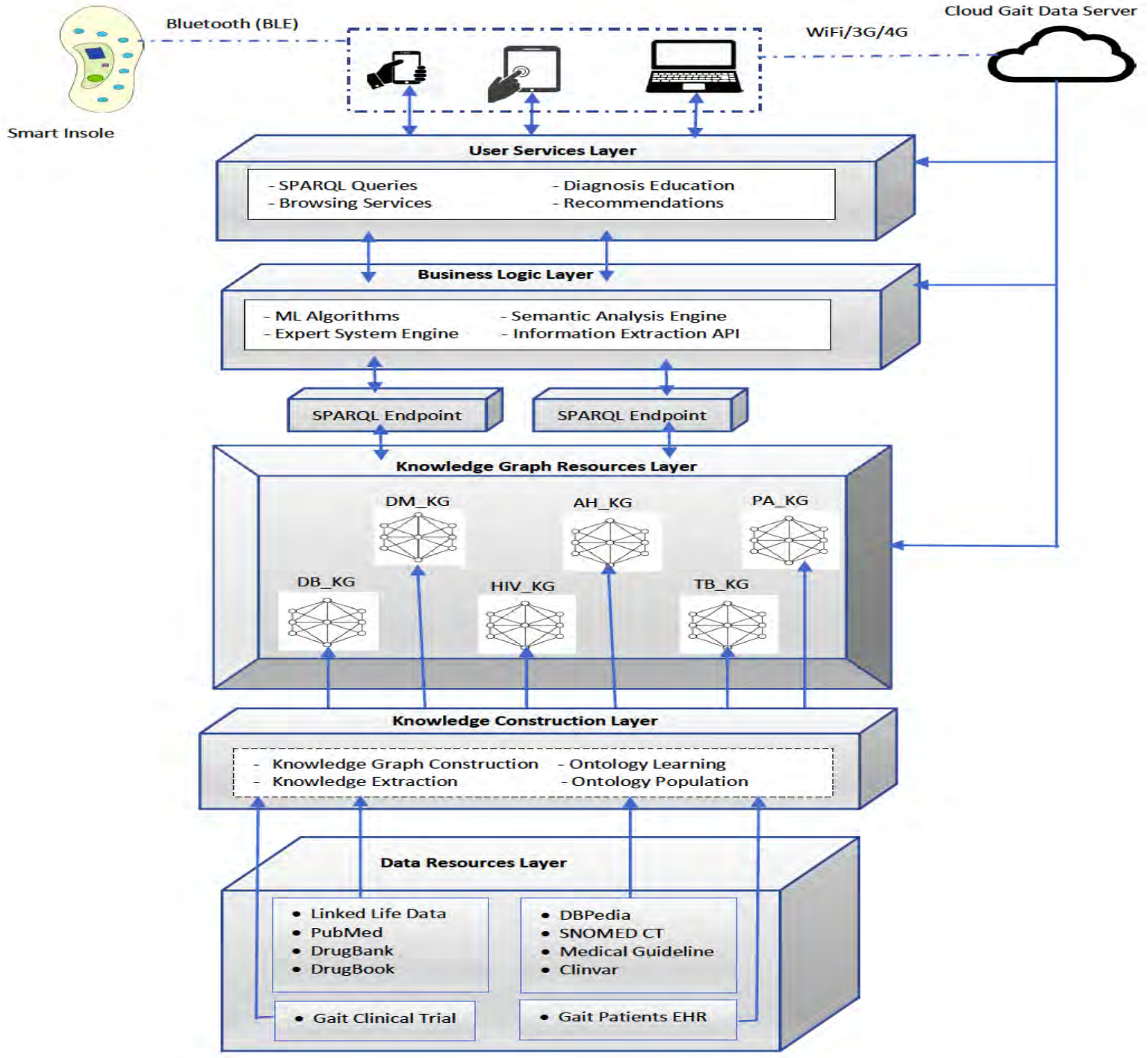

Figure 1. SemTreat Architecture Diagram

The SemTreat architecture (see Figure 1) is composed of four layers, which are described as follows:

User services Layer: this layer presents a user with opportunities to access the various services that the SemTreat architecture affords. This includes search and query services, recommendations, health education, and diagnosis.

Business Logic Layer: this layer enables the SemTreat architecture to construct an appropriate response to a user's query in a timely manner. The various algorithms, and computational modules that performs machine-learning-based prediction based on data, expert systems diagnosis, recommendations on health and wellness requests from users, and semantic analysis are contained in this layer. The components of this layer function based on raw data and semantic web data that are retrieved by using SPARQL endpoints in form of RDF triples from specific knowledge graphs (KG) in the lower level of the SemTreat architecture.

Knowledge Graph Resources Layer: this layer is composed of different knowledge graphs that have been pre-constructed through an offline process for specific types of diseases such as tuberculosis (TB_KG), HIV AIDS (HIV_KG), Diabetes (DB_KG), Dementia (DM_KG), Alzheimer's Disease, Parkinson's Disease. 
Knowledge Graph Construction Layer: this layer subsumes the myriad of offline activities that enable the construction of relevant knowledge graphs (KG) from multiple health data sources. Key operations that are deployed at this level include ontology learning, ontology population, and information extraction from raw health data sources. The knowledge graphs are constructed from aggregating data from diverse relevant open sources in order to build a rich data pool for specific diseases. In order to build/construct the KG for a particular disease, the clinical gait data, and electronic health records of gait patients of that particular disease, and relevant epidemiological data are used to compose the data pool for that specific disease.

Data Resources Layer: this is a suite of open health data resources, and sources of textual medical knowledge (TMK) that provide good basis for generating a credible knowledgebase for specific types of diseases. The knowledge graph construction entails extracting relevant knowledge from these sources, ensuring their semantic integration in form of knowledge graphs in a way that can be used by web services and computational modules at run-time for decision making. A description of some exemplary medical knowledge sources from which data could be extracted is shown in Table 1.

Table 1. Medical Knowledge Sources to be integrated into the SemTreat framework

\begin{tabular}{|l|l|l|l|}
\hline S/n & Sources & Description & \\
\hline 1 & $\begin{array}{l}\text { Linked Life Data } \\
\text { (LLD) }\end{array}$ & $\begin{array}{l}\text { LLD as a data-as-a-service platform } \\
\text { enables 25 public biomedical databases to } \\
\text { be accessed through a single access point }\end{array}$ & http://inkedlifedata.com/ \\
\hline 2 & PubMed & $\begin{array}{l}\text { PubMed contains over 29 million open } \\
\text { access articles from biomedical literature. }\end{array}$ & https://www.ncbi.nlm.nih.gov/pubmed/ \\
\hline 3 & DrugBank & $\begin{array}{l}\text { This database contains information on } \\
\text { drug data and drug targets. It can be } \\
\text { accessed using an API. }\end{array}$ & https://www.drugbank.ca/ \\
\hline 4 & DrugBook & $\begin{array}{l}\text { This is an e-book that offers rich textual } \\
\text { knowledge on drugs }\end{array}$ & \\
\hline 5 & DBPedia & $\begin{array}{l}\text { This exposes data in Wikipedia in form of } \\
\text { a database for open access }\end{array}$ & https://wiki.dbpedia.org/ \\
\hline 6 & SNOMED CT & $\begin{array}{l}\text { This is a comprehensive vocabulary of } \\
\text { clinical healthcare terminology. }\end{array}$ & http://www.snomed.org/ \\
\hline 7 & Clinvar & $\begin{array}{l}\text { this provides access to information about } \\
\text { the relationships asserted between human } \\
\text { variation and observed health status, and } \\
\text { the history of that interpretation. }\end{array}$ & https://www.ncbi.nlm.nih.gov/clinvar/ \\
\hline
\end{tabular}

\subsection{SemTreat Framework Use Case Scenarios}

In this section, we present few use cases of the SemTreat framework as relevant to a patient, or a doctor by using exemplar narratives.

Case 1. Mr X, is a 70 years old male with gait impairment due to Tuberculosis (TB) ailment. $X$ wants to receive recommendation on suitable recreational activities as a form self-management that can aid his wellness. As a result of wearing a smart insole for a period of 3 months, gait data such as his cadence, gait speed, step length, and stride length was constantly monitored and recorded. Since X is domiciled in a low-resource setting, gait data were temporarily stored in his mobile device from time to time before they are subsequently transferred to the cloud via $\mathrm{WiFi}$ or mobile $(3 \mathrm{G} / 4 \mathrm{G})$ connectivity. When $\mathrm{X}$ seeks recommendation to be advised on the type of recreational activities that could help to improve his personal fitness, the recommendation for $\mathrm{Mr} \mathrm{X}$, will based on the level of severity of TB ailment as inferred from the stored gait patterns that are inherent in his gait data, and the diverse forms of knowledge about TB that are stored in TB KG. This will include using the documentation of TB treatment cases, clinical trials, epidemiological data, and TB knowledge from diverse sources as contained in the TB knowledge graph, and the EHR of Mr X in order to give him an appropriate recommendation. 
Case 2. Dr $\mathrm{Y}$ is an expert in the treatment of Type II Diabetes and needs to respond to a request for advice from Patient B, female, who is Type II Diabetes Patient that is personally know by him, but who is currently in a remote location. The severity of Patient B's ailment has affected her gait, and such as worn the smart insole for the purpose of real-time gait analysis for 2 months. Dr Y wants to use the expert diagnosis service of the SemTreat framework as decision support tool in order to attend to Patient's B query. The request from Patient $B$ is that she has witnessed some symptoms lately that appear strange and needs to know what is going on. The request from Patient $B$ as any other request of the same nature is uniquely identified by the unique medical record number/medical insurance number of Patient B. Dr Y needs to access the electronic health record of Patient B in order to know her medical history. An encrypted message from Dr Y asking Patient B to give consent/permission to Dr Y to access her medical records is sent to Patient B. Patient B must give an approval/ consent before access is granted to Dr Y. Once the permission to access personal EHR is granted by Patient B, her pre-stored gait data also becomes available for the SemTreat algorithms to use in order to construct a medical advice on Patient B for the consideration of Dr Y. By using the combination of relevant extracted knowledge from the Diabetes KG, Gait data, and EHR of Patient B, Dr Y will receive machine learning-based decision support on how to respond to the request from Patient $\mathrm{B}$.

\section{EVALUATION CONCEPT}

The evaluation concept for the SemTreat framework will be guided by two perspectives. The first perspective of the evaluation will focus on performance and usability. For this, the emphasis will be on the atomic/individual user-based services that the SemTreat framework affords to assess their performance and usability. The services, such as expert diagnosis, health recommender system, and information search shall be experimentally evaluated by using standard performance metrics such as FI, recall, precision, and accuracy. Also, the usability of the user-based services shall be assessed both within and outside the clinic environment to determine users' perceptions of the services. The usability evaluation shall be done mainly through qualitative evaluation by user experiments feedbacks, questionnaire surveys, interviews and case studies. The second perspective of the evaluation is to assess the practicability and effectiveness of the framework in terms of its support for treatment decision-making for selected gait-related diseases from the viewpoints of patients/users, and medical practitioners. The emphasis of this particular evaluation is to determine whether the framework can indeed attain its envisioned goals for low-resource settings and can be integrated effectively into the operational workflow of medical practitioners for use, and also the daily lifestyle of an average user/patient. To do this, the Goal Question Metric (GQM) paradigm (Basili et al., 1994) will be used to conduct a holistic evaluation of the framework in order to assess the extent to which it can attain the goals and objectives that are associated with providing support for treatment decision making in low resource settings.

\section{CONCLUSION AND FUTURE WORK}

In this paper, we have presented the description of a conceptual framework ((SemTreat) that can enable the sematic integration of multiple health data sources for treatment decision-making for gait-related diseases in low-resource settings. The framework affords a suite of user-based services that aid the decision-making process of medical practitioners and help users/patients in the area of self-management for healthy living and wellness. The SemTreat framework is presented as capable of leveraging aggregated sematic knowledge from diverse sources to deliver intelligent services such as search and browsing, health recommendations, and expert disease diagnosis, while providing support for low cost and affordable technologies for real-time gait analysis.

However, the eventual realization of the SemTreat framework depends on giving attention to some additional non-functions requirements that will ensure the overall success of the framework. This includes the following: 
- Security: dealing with health information require that all data must be treated with security by ensuring that that personal data of patients are protected, and the data privacy is not compromised

- Accessibility: the framework must provide easy ways for people to assess his resources

- Cost: there must be emphasis on the use for low-cost technologies that are affordable for poor people. This include the use of ensuring that the design and product development is suitable for.

In future work, we will prototypically implement the components/subsystems that make up the SemTreat framework, so that we can proceed with the evaluation.

\section{ACKNOWLEDGEMENT}

This work was supported by the Centre for International Cooperation \& Mobility (ICM) of the Austrian Agency for International Cooperation in Education and Research (OeAD-GmbH) under Grant No. ZA 10/2019. It is also supported by the National Research Foundation of South Africa under Grant No. STGR 180414320796.

\section{REFERENCES}

Adebesin, F., Foster, R., Kotzé, P., Van Greunen, D., 2013. A Review of Interoperability Standards in E-health and Imperatives for their Adoption in Africa. South African Computer Journal 50. https://doi.org/10.18489/sacj.v50i1.176

Adenuga, O.A., Kekwaletswe, R.M., Coleman, A., 2015. eHealth integration and interoperability issues: towards a solution through enterprise architecture. Health Information Science and Systems 3. https://doi.org/10.1186/s13755015-0009-7

Aldred, L., Aalst, W.M.P., Dumas, M., Ter Hofstede, A., 2006. Understanding the challenges in getting together: The semantics of decoupling in middleware.

Asaria, M., Grasic, K., Walker, S., 2016. Using Linked Electronic Health Records to Estimate Healthcare Costs: Key Challenges and Opportunities. PharmacoEconomics 34, 155-160. https://doi.org/10.1007/s40273-015-0358-8

Basili, V.R., Caldiera, G., Rombach, H.D., 1994. The Goal Question Metric Approach, in: Encyclopedia of Software Engineering. Wiley.

Doan, A., Halevy, A.Y., 2005. Semantic-integration research in the database community. AI Mag. 26, 83-94.

Effenberg, A., Fehse, U., Weber, A., 2011. Movement Sonification: Audiovisual benefits on motor learning. BIO Web of Conferences 1, 00022. https://doi.org/10.1051/bioconf/20110100022

Fonseca, M., Karkaletsis, K., Cruz, I.A., Berler,. A., Oliveira, I.C., 2015. OpenNCP: a novel framework to foster cross-border e-Health services. Studies in Health Technology and Informatics 617-621. https://doi.org/10.3233/978$1-61499-512-8-617$

Fritz, F., Tilahun, B., Dugas, M., 2015. Success criteria for electronic medical record implementations in low-resource settings: a systematic review. Journal of the American Medical Informatics Association 22, 479-488. https://doi.org/10.1093/jamia/ocu038

Hohpe, G., 2006. Conversation Patterns: Workshop Report. Dagstuhl Seminar 7.

Horak, F., King, L., Mancini, M., 2015. Role of Body-Worn Movement Monitor Technology for Balance and Gait Rehabilitation. Physical Therapy 95, 461-470. https://doi.org/10.2522/ptj.20140253

Horsak, B., Dlapka, R., Iber, M., Gorgas, A.-M., Kiselka, A., Gradl, C., Siragy, T., Doppler, J., 2016. SONIGait: a wireless instrumented insole device for real-time sonification of gait. Journal on Multimodal User Interfaces 10, 195-206. https://doi.org/10.1007/s12193-016-0216-9

Lobo, J.M., Denton, B.T., Wilson, J.R., Shah, N.D., Smith, S.A., 2017. Using claims data linked with electronic health records to monitor and improve adherence to medication. IISE Transactions on Healthcare Systems Engineering 7 , 194-214. https://doi.org/10.1080/24725579.2017.1346728

Martinez-Costa, C., Legaz-Garcia, M.C., Schulz, S., Fernandez-Breis, J.T., 2014. Ontology-based infrastructure for a meaningful EHR representation and use, in: IEEE-EMBS International Conference on Biomedical and Health Informatics (BHI). Presented at the 2014 IEEE-EMBS International Conference on Biomedical and Health Informatics (BHI), IEEE, Valencia, Spain, pp. 535-538. https://doi.org/10.1109/BHI.2014.6864420

Morley, K.I., Wallace, J., Denaxas, S.C., Hunter, R.J., Patel, R.S., Perel, P., Shah, A.D., Timmis, A.D., Schilling, R.J., Hemingway, H., 2014. Defining Disease Phenotypes Using National Linked Electronic Health Records: A Case Study of Atrial Fibrillation. PLoS ONE 9, e110900. https://doi.org/10.1371/journal.pone.0110900 
Moser, T., Biffl, S., 2012. Semantic Integration of Software and Systems Engineering Environments. IEEE Transactions on Systems, Man, and Cybernetics, Part C (Applications and Reviews) 42, 38-50. https://doi.org/10.1109/TSMCC.2011.2136377

Noehren, B., Scholz, J., Davis, I., 2011. The effect of real-time gait retraining on hip kinematics, pain and function in subjects with patellofemoral pain syndrome. British Journal of Sports Medicine 45, 691-696. https://doi.org/10.1136/bjsm.2009.069112

Noy, N.F., 2004. Semantic integration: a survey of ontology-based approaches. ACM SIGMOD Record 33, 65. https://doi.org/10.1145/1041410.1041421

Redd, C.B., Bamberg, S.J.M., 2012. A Wireless Sensory Feedback Device for Real-Time Gait Feedback and Training. IEEE/ASME Transactions on Mechatronics 17, 425-433. https://doi.org/10.1109/TMECH.2012.2189014

Rodger, M.W.M., Young, W.R., Craig, C.M., 2014. Synthesis of Walking Sounds for Alleviating Gait Disturbances in Parkinson's Disease. IEEE Transactions on Neural Systems and Rehabilitation Engineering 22, 543-548. https://doi.org/10.1109/TNSRE.2013.2285410

Tang, W., Su, D., 2013. Locomotion analysis and its applications in neurological disorders detection: state-of-art review. Network Modeling Analysis in Health Informatics and Bioinformatics 2, 1-12. https://doi.org/10.1007/s13721-0120020-8

Zargaran, E., Schuurman, N., Nicol, A.J., Matzopoulos, R., Cinnamon, J., Taulu, T., Ricker, B., Garbutt Brown, D.R., Navsaria, P., Hameed, S.M., 2014. The Electronic Trauma Health Record: Design and Usability of a Novel Tablet-Based Tool for Trauma Care and Injury Surveillance in Low Resource Settings. Journal of the American College of Surgeons 218, 41-50. https://doi.org/10.1016/j.jamcollsurg.2013.10.001

Zhao, D., Weng, C., 2011. Combining PubMed knowledge and EHR data to develop a weighted bayesian network for pancreatic cancer prediction. Journal of Biomedical Informatics 44, 859-868. https://doi.org/10.1016/j.jbi.2011.05.004

Zhou, Y., Qureshi, R., Sacan, A., 2012. Data simulation and regulatory network reconstruction from time-series microarray data using stepwise multiple linear regression. Network Modeling Analysis in Health Informatics and Bioinformatics 1, 3-17. https://doi.org/10.1007/s13721-012-0008-4

Zillner, S., Sonntag, D., 2012. Image metadata reasoning for improved clinical decision support. Network Modeling Analysis in Health Informatics and Bioinformatics 1, 37-46. https://doi.org/10.1007/s13721-012-0003-9 